Historic, Archive Document

Do not assume content reflects current scientific knowledge, policies, or practices. 

FRANK W. MELICK Spencer, Ind.

\section{Season 1930 GLADIOLUS PRICE LIST}

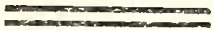

\section{Return Postage Guaranteed}
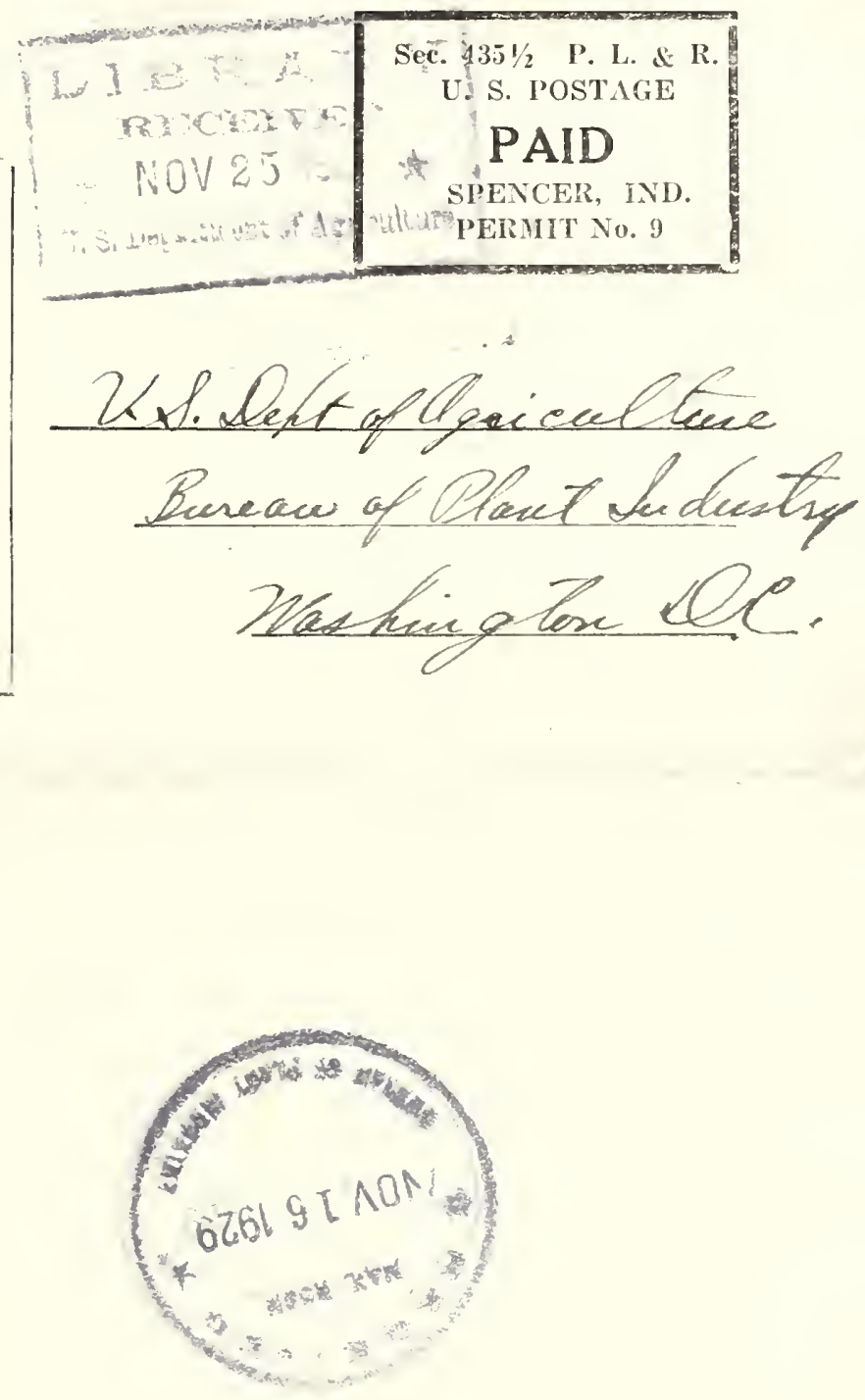

TERMS

CASH WITH ORDER PLEASE. No exira count on orders of less than $\$ 3.00$.

On order's of $\$ 5.00$ to $\$ 25.00$ besides the extra count we give, you may select extra bulbs amounting to 5 per cent of your order; 10 per cent on $\$ 25.00$ to $\$ 50.00$ orders; 15 per cent on $\$ 50.00$ to $\$ 100.00$ orders; and 20 per cent on $\$ 100.00$ and over. All orders sent postpaid or express prepaid anywhere in U. S. or Canada.

For reference write National Bank, Chamber of Commerce or Beaver Club, all of Spencer, Indiana.

MEMBER:-American Gladiolus Society, Indiana Gladiolus Society, Wabash Valley Gladiolus Society and Indiana State Florists' Association.

\section{DON'T DELAY}

Send us your order at once. Many varieties will soon be sold out at these prices. We will, unless notified otherwise by you, substitute other sizes of the same variety if out of size ordered, but we da not substitute other varieties unless you instruct us to do so. 
A. E. KUNDRED-large ruffled salmon rose ALBANIA-best medium priced white ALLEN V. BUNCE-vivid orange yellow ANNA EBERIUS-velvety purple BREAK O' DAY - very early pink CARMEN SYLVA-good tall white CRIMSON GLOW-glowing crimson CROWN OF GOLD _-light yellow

DR. F. E. BENNETT-"House Afire" red ELIZABETH SWARTLEY-early pink FAY LANPHIER - cerise FORT SUMNER-fine scarlet GEORGE J. JOERG-sa'mon pink GERALDINE FARRAR-lavender violet GERTRUDE ERIEY-shell pink GIANT NYMPH-La France pink GLADDIE BOY-rose pink GOLD-best light yellow GOLD EAGLE-early golden yellow GOLDEN DREAM-tall deep yellow GOLDEN SWALLOW-clear ruffled yellow IIALLEY-standard early salmon p ink HARBINGER — orange red HEAVENLY BLUE-lavender blue HELEN HOWARD-buff yellow HELEN PHIPPS-deep pink JOERG'S WHITE-mammoth white JUNIATA-pink, white throat LONGFELLOW-tall pink LOS ANGELES-grenadine pink LUTHER BURBANK (K)-geranium red MARIE KUNDRED-best early white MARY FREY-lavendar MARY JANE-lavender pink MEMORY OF WM. PFI'TER-large white MING TOY-buff yel'ow

MINUET-fine lavender

MISS DEMURE-bluch white MOTHER MACREE-best of the smoke shades MRS. F. C. HORNBERGER-good white MRS. FRANK PENDLETON-rose pink MRS. H. E. BOTHIN-ruffled geranium pink MRS. LEO E. MILLER-fine salmon MRS. LEON DOUGLAS-tall salmon pink MRS. P. W. SISSON-large cameo pink MR. W. H. PHIPPS-large pink 1910-ROSE-deep rose pink ORANGE WONDER-very good ORCHID LADY-archid lavender PATRICIA CARTER-early shrimp pink PEACE-tall white, blotched red PEARL OF CALIFORNIA-LaFrance pink PFITZERS TRIUMPH-salmon orange PURPLE GLORY-deep maroon RADIO-rhodonite pink RED TORNADO-very brilliant red RUFFOLACE-ruffed creamy white THE ARISTOCRAT-saffron pink THE ORCHID-lacinated lavender pink TYCKO ZANG-large salmon pink WHITE PREMEIR-good white YELLOW WONDER-deep yellow

\begin{tabular}{|c|c|c|c|c|c|}
\hline per & over & $\begin{array}{l}\frac{8}{4} \text {-in. } \\
\text { to } 1 \frac{1}{4}\end{array}$ & \& under & & \\
\hline & .40 & .25 & .15 & 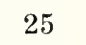 & \\
\hline & .50 & .25 & .15 & 100 & .10 \\
\hline & .20 & & a & 25 & .25 \\
\hline & .50 & & & & \\
\hline & 1.00 & .50 & .30 & 100 & .25 \\
\hline & .50 & & .15 & 100 & .15 \\
\hline & .50 & & 0 & 100 & .15 \\
\hline & .75 & 40 & .25 & 25 & .25 \\
\hline & 1.00 & & .25 & 100 & .50 \\
\hline & .50 & & 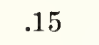 & 25 & .25 \\
\hline & 1.00 & .75 & .50 & 100 & .50 \\
\hline & 2.00 & $\because$ & $\cdots$ & $\cdots$ & \\
\hline & .75 & . & $\ldots$ & .. & \\
\hline & .30 & $\ldots$ & $\ldots$ & ... & .. \\
\hline & 1.50 & 1.00 & .70 & 100 & .50 \\
\hline & .50 & & $\ldots$ & ... & \\
\hline & .50 & 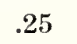 & 15 & 25 & .20 \\
\hline & .60 & .40 & .20 & 100 & .2 \\
\hline 1 & .20 & .1 & .10 & 20 & .2 \\
\hline & .35 & & & 1 & .2 \\
\hline & .50 & & & 5 & .1 \\
\hline & .5 & .3 & .20 & 250 & .2 \\
\hline$x^{-1}$ & .50 & .30 & .20 & 25 & .5 \\
\hline 1 & 1.50 & 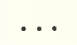 & . & ... & $\cdots$ \\
\hline 1 & .50 & & $\ldots$ & 12 & .5 \\
\hline 1 & 1.50 & 00 & .75 & & .1 \\
\hline 3 & 1.00 & & $\ldots$ & ... & . \\
\hline 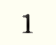 & .75 & .b) & .25 & 12 & .5 \\
\hline & 7 & & 2 & 5 & .2 \\
\hline & .60 & .3 & .20 & 25 & .1 \\
\hline 1 & 2.00 & & $\ldots$ & . & 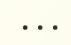 \\
\hline 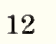 & .75 & & & 25 & .10 \\
\hline 1 & .75 & .50 & .25 & 6 & .2 \\
\hline 1 & .75 & .50 & 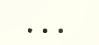 & 6 - & .2 \\
\hline 1 & & & 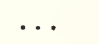 & $\cdots$ & \\
\hline & .50 & 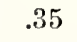 & .20 & 50 & .25 \\
\hline 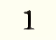 & .7 & 5 & & 10 & .5 \\
\hline 6 & 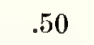 & 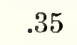 & .20 & 25 & .2 \\
\hline & 25.00 & & 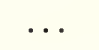 & 1 & 1.0 \\
\hline 1 & & & & . & $\cdots$ \\
\hline & & & & & 2 \\
\hline & .50 & .35 & & 50 & .25 \\
\hline 1 & $\ldots$ & $\ldots$ & 0 & 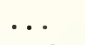 & \\
\hline & & & & 100 & .2 \\
\hline 1 & & & & & 2 \\
\hline & 1.60 & 00 & 0 & 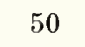 & .25 \\
\hline & & & .15 & 250 & .2 \\
\hline 1 & & & 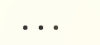 & . & 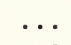 \\
\hline 1 & 75 & 0 & .25 & 12 & .5 \\
\hline 1 & & & $\cdots$ & . & $\cdots$ \\
\hline 2 & .50 & 5 & 15 & 250 & .2 \\
\hline 1 & .75 & ... & . & $\ldots$ & \\
\hline 9 & .7 & & & $\ldots$ & \\
\hline 6 & .5 & & $\cdots$ & $\ldots$ & \\
\hline 1 & & & .25 & 12 & .00 \\
\hline 1 & & 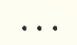 & ... & ... & . \\
\hline 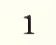 & & & & 12 & .5 \\
\hline 3 & & & .20 & 25 & .2 \\
\hline 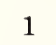 & & & .10 & 25 & .2 \\
\hline to & & & .25 & 150 & .2 \\
\hline 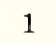 & & & .10 & 25 & .2 \\
\hline & 25 & .15 & .10 & 25 & 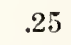 \\
\hline
\end{tabular}

